\title{
Simulación del compensador estático de VAR de la subestación Culiacán Tres para el análisis de transitorios
}

\section{Simulation of the Static VAR Compensator Culiacan Three Substation for the Analysis of Transients}

Coronel-Mercado Luis Enrique

Comisión Federal de Electricidad

Gerencia Regional de Transmisión Noroeste

Zona de Transmisión Culiacán, México

Correo:luis.coronelo1@cfe.gob.mx

\author{
Huerta-González Pedro Francisco \\ Ingeniería en Control y Automatización \\ Escuela Superior de Ingeniería Mecánica y Eléctrica \\ ESIME-Zacatenco, Instituto Politécnico Nacional, México \\ Correo:phuerta@ipn.mx
}

Rodríguez-Rivas Jaime José

Sección de Estudios de Posgrado e Investigación

Escuela Superior de Ingeniería Mecánica y Eléctrica

ESIME-Zacatenco, Instituto Politécnico Nacional, México

Correo: jjrodriguezr@ipn.mx

Información del artículo: recibido: mayo de 2012, reevaluado: julio de 2012, aceptado: septiembre de 2012

\section{Resumen}

Desde su aparición, a finales de la década de los 60, los sistemas FACTS (flexible AC transmision systems) han sido y son estudiados e implementados en todas las redes eléctricas alrededor del mundo, convirtiéndose en un elemento esencial para la estabilidad, control y máxima explotación de las mismas. Uno de los dispositivos FACTS más utilizados es el Compensador Estático de VAR o CEV. En este trabajo se ha realizado el modelado en Simulink del CEV, instalado en la subestación Culiacán Tres (CUT) de la CFE. Este CEV es de la marca ABB con capacidad de \pm 100 MVAR. El modelado se realizó a detalle, programando visualmente en Simulink los diferentes subsistemas que forman el CEV como: la unidad de sincronía, el TSC (capacitor conmutado por tiristores), el TCR (reactor controlado por tiristores), los filtros de 5 a y 7a armónica, el banco de transformadores, la unidad de control, la unidad de distribución y la unidad de medición. Los datos para los principales componentes se tomaron de los manuales y reportes técnicos del fabricante del equipo. Se utilizó el programa ASPEN con el objetivo de obtener equivalentes de Thevenin del SEP (sistema eléctrico de potencia) y conectar a ellos el modelo del CEV con el objetivo de realizar pruebas de respuesta al escalón y la respuesta del CEV ante un corto circuito trifásico; los resultados se comparan con resultados obtenidos en el PSS/E y con resultados de las pruebas realizadas al CEV durante la puesta en servicio.

\section{Descriptores:}

- compensador estático de VAR

- modelado

- simulación del CEV 


\begin{abstract}
Since its emergence in the late 60's the FACTS (Flexible AC Transmission Systems) have been and continue to be studied and implemented in all electrical networks around the world, becoming an essential element for stability, control and maximum exploitation of thereof. One of the most used FACTS devices is the Static VAR Compensator or SVC. In this work, the modeling in Simulink of the Static VAR Compensator installed at the substation Culiacán Tres (CUT) belonging to CFE is presented. The SVC is the ability of \pm 100 MVAR and ABB brand. The modeling was performed to visually program in detail the different subsystems in Simulink such as the timing unit, the TSC (thyristor switched capacitor), TCR (thyristor controlled reactors), filters of the 5th and the 7 th harmonic, the transformer bank, the control unit, distribution unit and the measurement unit. Data for major components were taken from data sheet and equipment manuals. ASPEN software was used in order to obtain Thevenin equivalent of the EPS to connect to them the SVC model to test the step and three-phase short circuit. The results of these tests were compared with test results of commissioning of the SVC.
\end{abstract}

Keywords:

- static VARS compensator

- modeling

- SVC simulation

\section{Introducción}

Uno de los dispositivos FACTS (flexible AC transmisión systems) más importantes es el CEV o compensador estático de VAR, el cual puede ser utilizado para el control del voltaje y mejorar la estabilidad de los Sistemas Eléctricos de Potencia (SEP). Un CEV puede aportar la potencia reactiva necesaria para el control dinámico del voltaje, compensando así los desvíos de potencia reactiva de la red, provocados por grandes variaciones de carga, que podrían provocar variaciones inaceptables de voltaje, inestabilidades o hasta colapso del sistema (Braegger, 2005). El CEV también es usado para amortiguar las oscilaciones de potencia, mejorar la estabilidad transitoria y reducir las pérdidas en el sistema por medio del control de la potencia reactiva.

En México, la instalación de los CEV se inició en la década de los 80 en la Comisión Federal de Electricidad (CFE). En la actualidad la CFE cuenta con 20 CEV instalados a lo largo y ancho del territorio nacional en las redes de $115 \mathrm{kV}, 230 \mathrm{kV}$ y $400 \mathrm{kV}$, siendo los principales proveedores ABB y SIEMENS. El compensador estático de VAR que se presenta en este trabajo, se encuentra instalado en la Subestación Culiacán Tres (CUT), en la ciudad de Culiacán, Sinaloa. En la figura 1 se muestra la conexión del CEV en la CUT y las subestaciones interconectadas a ella por medio de líneas de $230 \mathrm{kV}$.

Este trabajo tiene como objetivo realizar el modelado y simulación del CEV instalado en la subestación Culiacán Tres (CUT) utilizando Simulink. El paquete Matlab/Simulink permite utilizar la librería de poten- cia: PowerLib, con lo cual se logra programar de forma gráfica (visualmente) todas las etapas de electrónica de potencia e insertarlas al sistema de potencia. Se utilizan los bloques del Simulink que modelan los elementos de una red como transformadores, líneas de transmisión y utilización de equivalentes del resto del sistema, con lo que se logra obtener un modelo del sistema en la zona de Culiacán, donde se encuentra conectado el CEV. En este trabajo el programa fue elaborado totalmente, y se ajusta lo más posible a las condiciones reales de operación del CEV de CUT, de tal forma que pueda ser utilizado para realizar estudios de operación dinámica y estática, que serán de gran utilidad, en particular, para el personal técnico que opera la subestación y en general para la CFE, ya que se tendrá una herramienta de análisis en donde se integren los elementos de electrónica de potencia sin que se consideren éstos como una caja negra, como en muchas ocasiones se ve en algunos simuladores. El programa, con futuras modificaciones y siguiendo la descripción expuesta en este trabajo, puede ser extendido a otros CEV del país.

La validación del modelo se hará con base en la comparación de los resultados de las simulaciones realizadas con el comportamiento real del CEV, durante las pruebas de puesta en servicio, y al comparar algunos resultados con los de otros programas comerciales utilizados en este tipo de análisis, como el ASPEN y el PSS/E.

Otro objetivo del trabajo es obtener equivalentes del SEP que permitan realizar estudios de operación del CEV ante condiciones de cortocircuitos. 


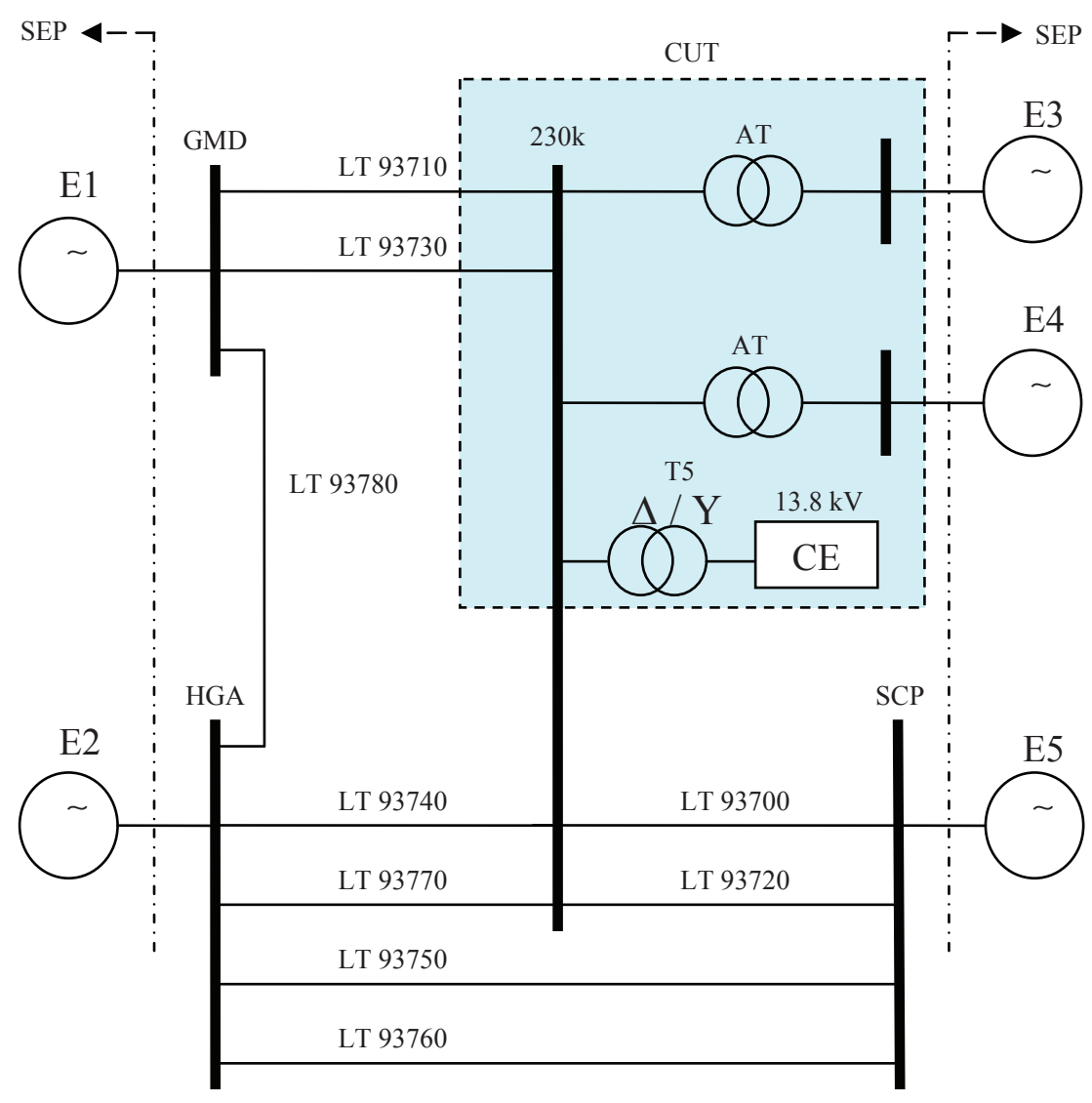

Figura 1. Diagrama unifilar de red troncal 230 kV de la zona Culiacán
El sistema de la figura 1 está formado por cuatro subestaciones de $230 \mathrm{kV}$, que son: subestación culiacán tres (CUT), subestación culiacán potencia (SCP), subestación higuera (HGA) y subestación guamúchil dos (GMD). Las líneas de transmisión se muestran con la nomenclatura normalizada por la CFE para líneas de $230 \mathrm{kV}$. Los elementos E1 a E5 de la figura 1 representan los equivalentes de Thevenin del sistema de potencia, más adelante se mencionan cuáles equivalentes de Thevenin se obtuvieron.

\section{Compensador estático de VAR (CEV)}

El CEV instalado en la subestación CUT está constituido fundamentalmente por un reactor controlado por tiristores (TCR), un capacitor conmutado con tiristores (TSC) y filtros de corrientes.

En el TCR el valor efectivo de la inductancia puede cambiar con la variación del ángulo de disparo en los tiristores. En la figura 2 se muestra la variación de la componente fundamental de la corriente en función del ángulo de disparo. Considerando que el voltaje en los terminales del TCR es constante, la variación de la co- rriente se puede interpretar como una variación de la reactancia y por lo tanto como una variación de la inductancia efectiva del reactor. Debido a la carga no lineal que representa el TCR para el sistema, se generan armónicos de corrientes que se requieren filtrar.

El valor de la susceptancia efectiva del reactor, $\mathrm{B}_{\mathrm{L}}(\alpha)$, en función del valor del ángulo de disparo $(\alpha)$ de los tiristores se puede calcular por la expresión (Hingorani et al., 2000):

$\mathrm{B}_{L}(\alpha)=\frac{1}{\omega \mathrm{L}}\left(1-\frac{2}{\pi} \alpha-\frac{1}{\pi} \operatorname{sen} 2 \alpha\right)=\frac{\mathrm{I}_{L F}(\alpha)}{\mathrm{V}}$

La variación de la capacitancia en el CEV se realiza conectando y desconectando capacitores con un mínimo de transitorios electromagnéticos. La conexión y desconexión se realiza utilizando interruptores electrónicos formados por una configuración bidireccional de dos tiristores de igual forma a como se tiene en el TCR, pero con la diferencia de que en el TSC la configuración de los dos tiristores trabaja como interruptor y en el TCR como regulador, debido a que en el TSC los tiristores 

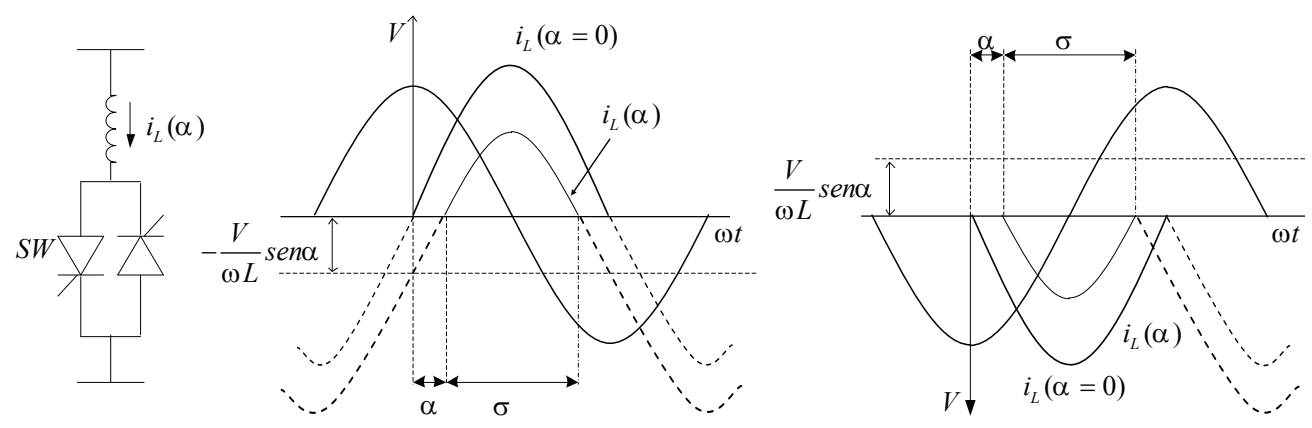

a)

b)

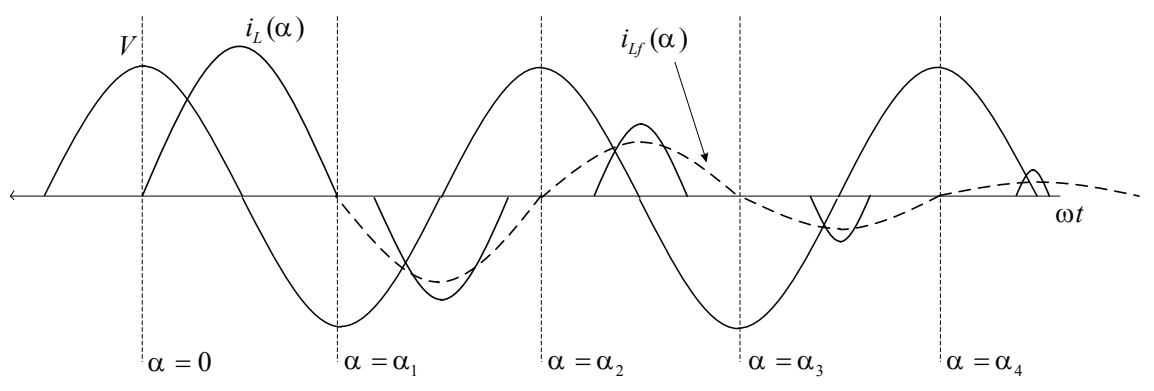

C)

Figura 2. a) reactor controlado por tiristor, b) control del ángulo de disparo, y c) formas de onda de operación (Hingorani et al., 2000)

realizan la función de un interruptor bidireccional, es decir, conectan y desconectan los capacitores en un determinado momento con el mismo valor de la capacitancia, sin embargo, en el TCR se controla el valor del ángulo de disparo para variar el valor de la inductancia efectiva del reactor y por lo tanto controlar la cantidad de reactivo que consume.

En la figura 3 se muestra un TSC monofásico que consiste en un capacitor, una válvula bidireccional de tiristores y un pequeño reactor limitador de corriente. En condiciones de estado estacionario, cuando la válvula de tiristores entra en conducción, el capacitor es conectado a la fuente de voltaje de CA, donde $v=V \operatorname{sen} \omega t$ y la corriente de la rama es (Hingorani $e t$ al., 2000):

$i(\omega t)=\mathrm{V} \frac{m^{2}}{m^{2}-1} \omega \mathrm{C} \cos (\omega t)$

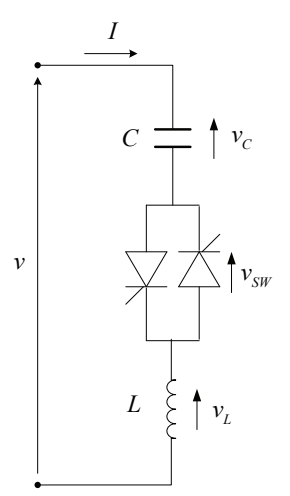

a)

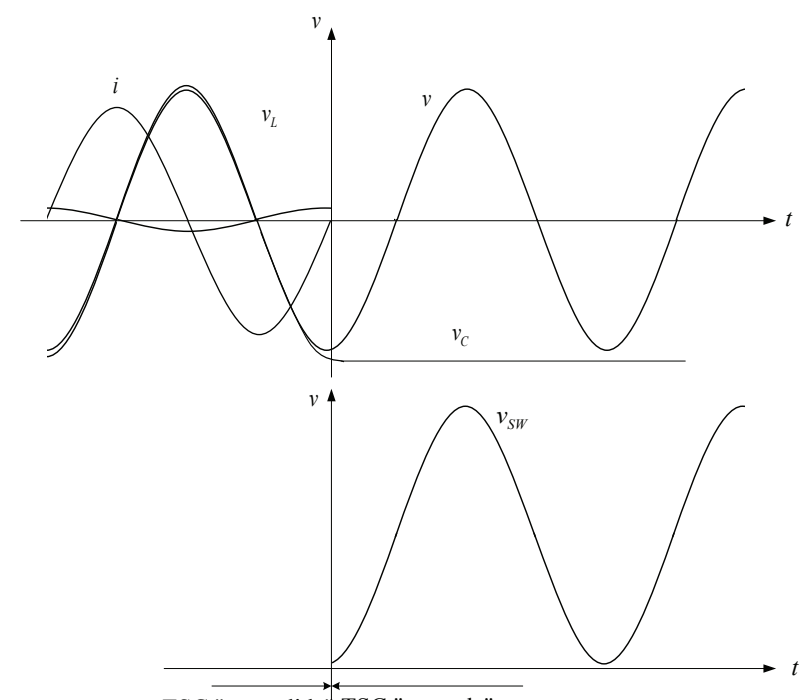

TSC "encendida"| TSC "apagada"

b)
Figura 3. a) configuración básica del capacitor conmutado por tiristores (TSC), y b) sus formas de onda (Hingorani et al., 2000) 
donde

$$
m=\frac{1}{\sqrt{\omega_{0}^{2} \mathrm{LC}}}=\sqrt{\frac{\mathrm{X}_{\mathrm{C}}}{\mathrm{X}_{\mathrm{L}}}}
$$

La frecuencia natural es:

$\omega_{n}=m \omega_{0}=\frac{1}{\sqrt{\mathrm{LC}}}$

donde $\omega_{0}=2 \pi f$, y $f$ es la frecuencia de la red.

La desconexión del capacitor se realiza de forma natural al dejar de aplicar pulsos de disparo a los tiristores. Para reducir al mínimo el transitorio en la reconexión del capacitor, el disparo de los tiristores se debe realizar cuando el voltaje en los terminales de la válvula de tiristores tiene un valor mínimo. En la figura 4 se muestran los transitorios para diferentes instantes de conexión del capacitor a la red de CA.

\section{Descripción general del CEV de CUT}

El CEV tiene un rango de operación continua de \pm 100 MVAR y está conectado en derivación a la red de 230 $\mathrm{kV}$ por medio de un banco de transformadores monofásicos (T5) con una potencia trifásica de 200MVA y un voltaje nominal de 230 / $13.8 \mathrm{kV}$. En la figura 5 se muestra el diagrama unifilar del CEV de CUT.

El CEV de CUT consiste en un banco de reactores controlado por tiristores (TCR) en configuración delta, un banco de capacitores conmutado por tiristores (TSC) en configuración estrella y dos bancos de capacitores fijos que funcionan como filtros de 5 a y 7a armónica. El sistema de control opera al CEV de modo que la potencia reactiva resultante pueda controlarse continuamente dentro del rango de máxima salida inductiva a máxima salida capacitiva. El objetivo principal del sistema de control es mantener el voltaje del bus de $230 \mathrm{kV}$ cercano a un punto de referencia de voltaje del sistema de control de voltaje del CEV.

La rama TSC del CEV de la subestación CUT (figura 5), tiene una potencia reactiva nominal de 50 MVAR capacitivos, y está formada por los siguientes elementos: válvula a tiristores (32 tiristores por fase formando 16 grupos de dos tiristores en antiparalelo conectados en serie, el valor máximo de la corriente por la válvula es de $2345 \mathrm{~A}$ ), banco de capacitores de $615.4 \mu \mathrm{F}$ por fase $\mathrm{y}$ reactor limitador de corriente de $0.598 \mathrm{mH}$ también por fase. A partir de la ecuación 4 se obtiene la frecuencia natural o frecuencia de sintonización $=262.3 \mathrm{~Hz}$ de la rama TSC, donde es la frecuencia fundamental del sistema. De la ecuación 3 el valor de $\mathrm{m}=4.37$ es adecuado,
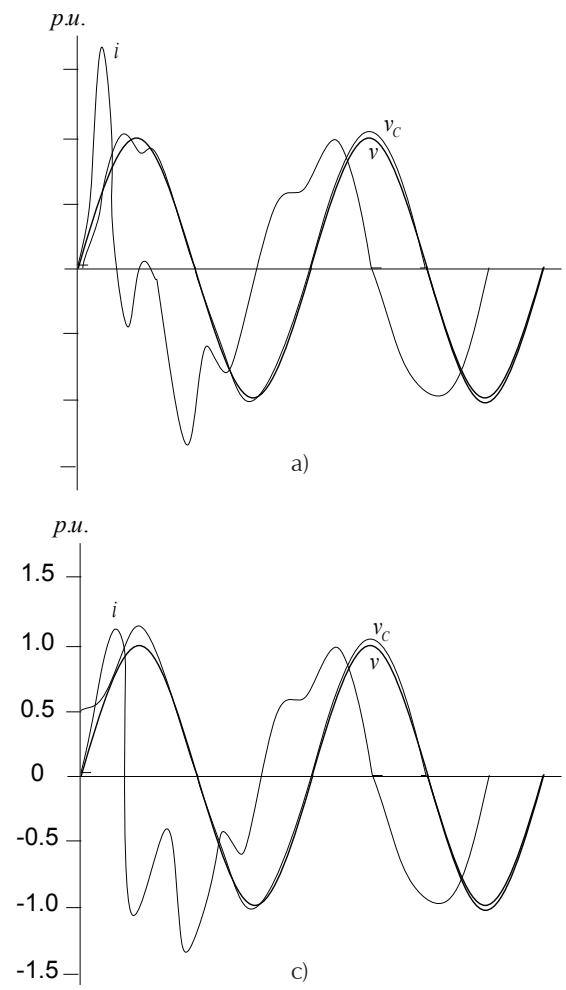
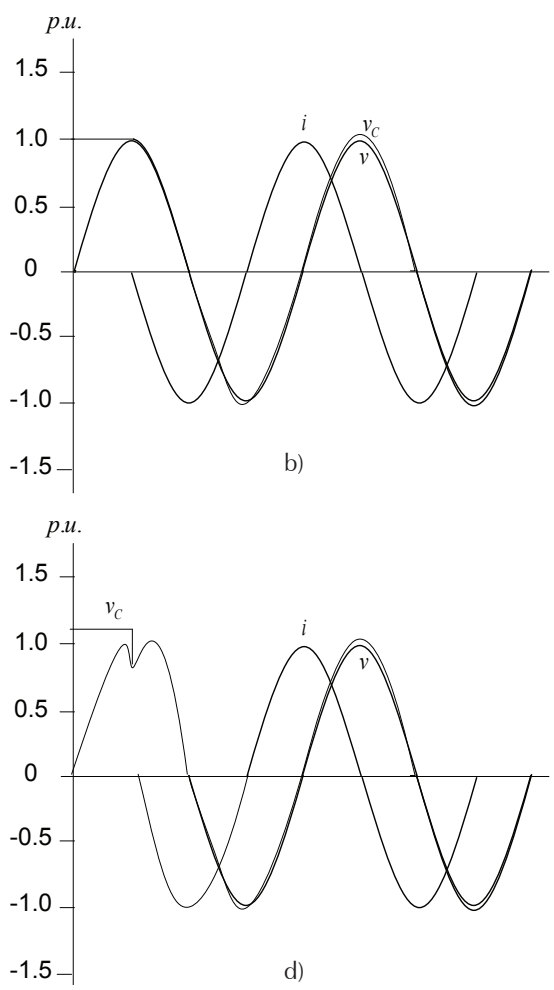

Figura 4. Estrategias de disparo en un TSC, a) disparo cuando el capacitor esta descargado, b) disparo cuando el capacitor tiene una carga inicial $\left.\left(\mathrm{v}_{\mathrm{c}}<\sqrt{2} \mathrm{~V}_{\mathrm{rms}}\right), \mathrm{c}\right)$ disparo cuando el capacitor tiene una carga inicial igual a la amplitud del voltaje aplicado $\left.\left(\mathrm{V}_{\mathrm{c}}=\sqrt{2} \mathrm{~V}_{\mathrm{rms}}\right), \mathrm{d}\right)$ disparo cuando el capacitor tiene una carga inicial mayor que la amplitud del voltaje aplicado $\left(\mathrm{v}_{\mathrm{c}}>\sqrt{2} \mathrm{~V}_{\mathrm{rms}}\right)$ (Hingorani et al., 2000) 


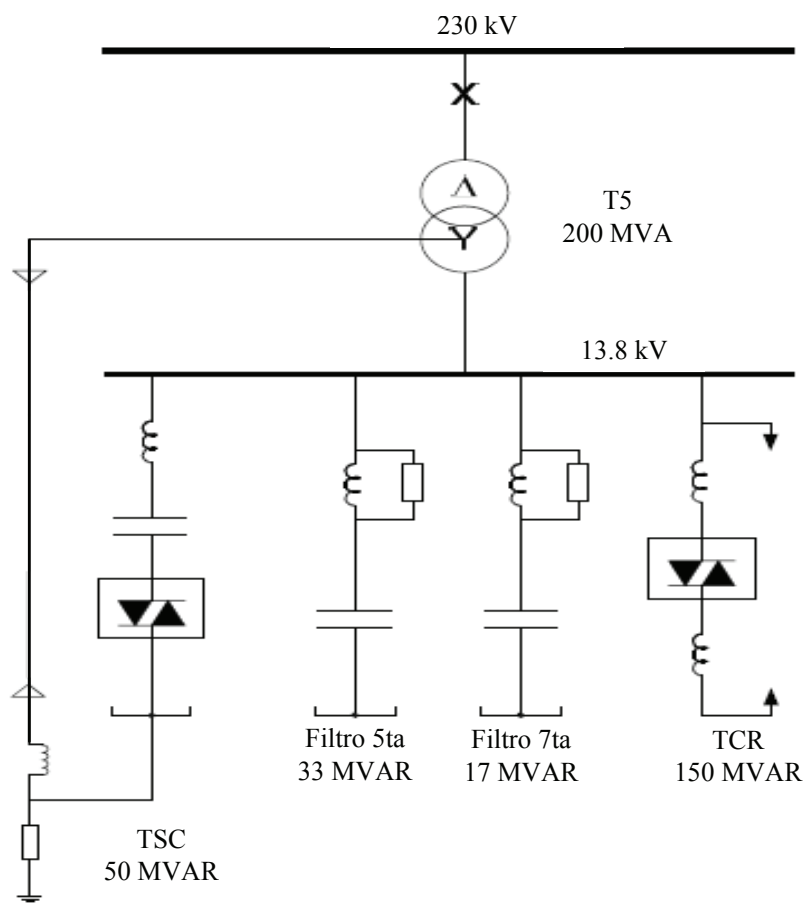

Figura 5. Diagrama unifilar del CEV de CUT

ya que en la práctica se toman valores entre 4 y 5 . La rama TCR del CEV de la subestación CUT, tiene una potencia reactiva nominal de 150 MVAR inductivos y está formada por el reactor y la válvula de tiristores en conexión delta con 20 tiristores formando 10 grupos de dos en antiparalelo, la inductancia por fase es de 9.59 $\mathrm{mH}$ y la corriente máxima a través de la válvula es de 4688 A.

Los filtros de 5a y 7a armónica tienen la función de evitar que las componentes armónicas de mayor magnitud generadas por el TCR pasen al lado primario del CEV e ingresen al sistema eléctrico de potencia. Para el filtro de 5 a armónica la frecuencia de resonancia es de $297 \mathrm{~Hz}$ y para el filtro de 7a armónica es de $417 \mathrm{~Hz}$. A la frecuencia fundamental del sistema de $60 \mathrm{~Hz}$, los filtros se comportan como capacitivos generando potencia reactiva capacitiva.

\section{Conexión TSC-TCR}

Para una regulación continua en todo su rango operativo es importante que al momento de la conexión y desconexión de la rama TSC se realice una compensación adecuada con la rama TCR. La figura 6 muestra las corrientes de las ramas TSC y TCR en función de la corriente total del CEV. El punto de conmutación A de la figura 6 es donde las susceptancias del TSC y del TCR son iguales, pero de signo contrario, por lo tanto se anulan entre ellas. En este punto de operación la susceptancia en el secundario del CEV corresponde a la susceptancia de los filtros de 5a y 7a armónica. Para el CEV de CUT el sistema de control está diseñado de tal manera que la desconexión de la rama TSC ocurra cuando la susceptancia del TCR sea mayor a 15\% (punto B de la figura 6) que la susceptancia del TSC (De Oliveira et al., 2001). En la figura 7 se muestra la variación de la susceptancia de referencia y el valor de $\alpha$ en la rama TCR durante la conexión y desconexión del TSC.

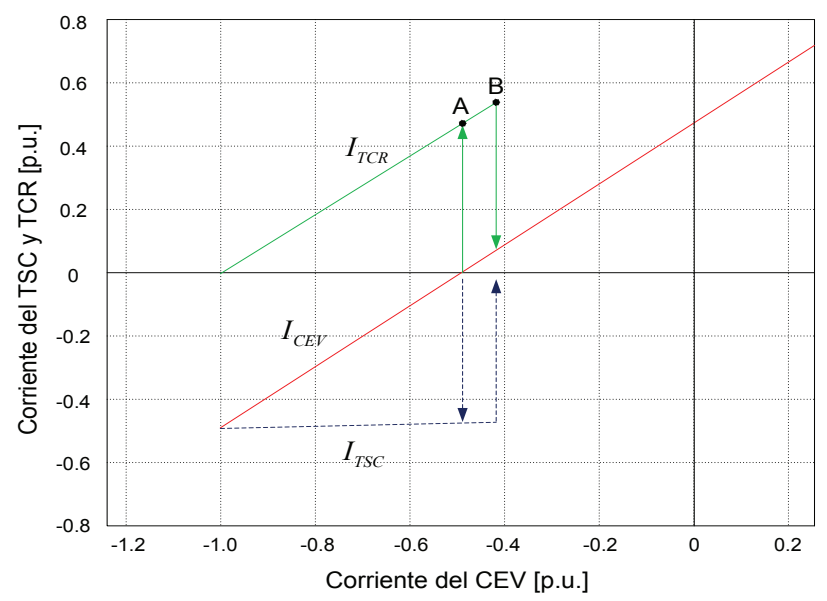

Figura 6. Puntos de conmutación del TSC a 1.0 p.u. del voltaje primario

\section{Sistema de sincronización y generación de pulsos de disparo hacia los tiristores}

El propósito del sistema de sincronización es generar pulsos de sincronismo con la componente fundamental de voltaje del sistema (Mohan et al., 2002). Entre los métodos disponibles se utilizó el lazo enganchado de fase (PLL), que es el método de sincronización más utilizado en HVDC y CEV. Una vez lograda la sincronización del circuito de disparo con las señales de los voltajes de fase, se pueden generar los pulsos de disparo de los tiristores. Para el disparo de los tiristores del TCR, se usa el método de control lineal de ángulo de disparo, el cual toma las señales de voltaje filtradas por el PLL, las convierte a señales cuadradas mediante detectores de cruce por cero para después integrarlas y generar dientes de sierra. La señales diente de sierra son comparadas con una señal de referencia y en el instante donde ambas señales son iguales, se genera la señal de disparo hacia los tiristores del TCR. Se utilizó un PLL en la simulación similar al utilizado por ABB en el CEV.

Las señales más importantes obtenidas con la programación gráfica en Simulink se muestran en la figura 8. 

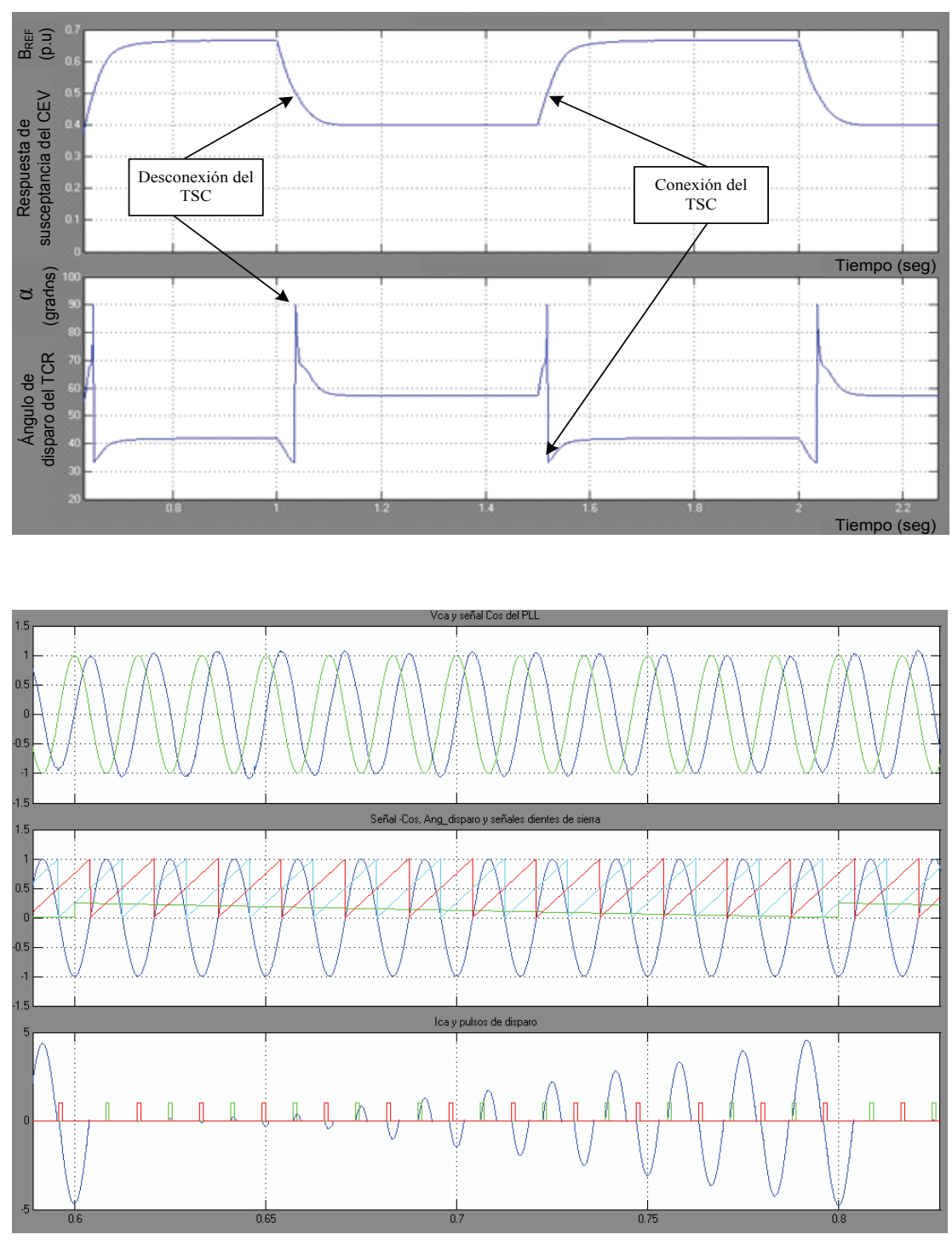

Figura 7. Control de $\alpha$ del TCR a la conexión y desconexión del del TSC

Figura 8. Formas de onda del bloque Sincronía
En esta figura se observan en la parte superior una señal de fase y la señal coseno generada por el PLL. En la figura del centro se muestran la señal -cos, la señales dientes de sierra generadas a partir de la señal -cos y la señal de referencia, la cual se colocó variable en el tiempo para que se observen las variaciones del ángulo de disparo de los tiristores. Por último, en la parte inferior se observan los pulsos de disparo y la señal de corriente por los reactores del TCR. Se puede apreciar que las formas de onda de estas señales coinciden con las mostradas en la figura 2.

En el TSC la sincronización de los pulsos de disparo para la conexión del capacitor se realizó tomando en cuenta el criterio de mínimo transitorio, tal y como se describió anteriormente en las figuras 3 y 4.

\section{Control automático de voltaje}

El propósito de este control es mantener el CEV de CUT funcionando con una característica V-I dada por los valores de voltaje de referencia y pendiente fijados (Pettersson et al., 2005) y señalados en la documentación de ABB. En Taylor et al. (1994) se estandarizan los modelos del control de CEV de donde se obtiene el diagrama a bloques del regulador de voltaje del CEV de CUT que se muestra en la figura 9.

La variable $V_{t}$ es el voltaje instantáneo del lado de alta de CEV, el cual entra al bloque del transductor, donde $\mathrm{T}_{\text {MEASURE }}$ es la constante de tiempo del transductor de voltaje. El transductor entrega una medición de voltaje $\mathrm{V}_{\mathrm{RESP}}$, la cual se resta en el punto suma. $\Delta \mathrm{V}_{\mathrm{REF}}$ 


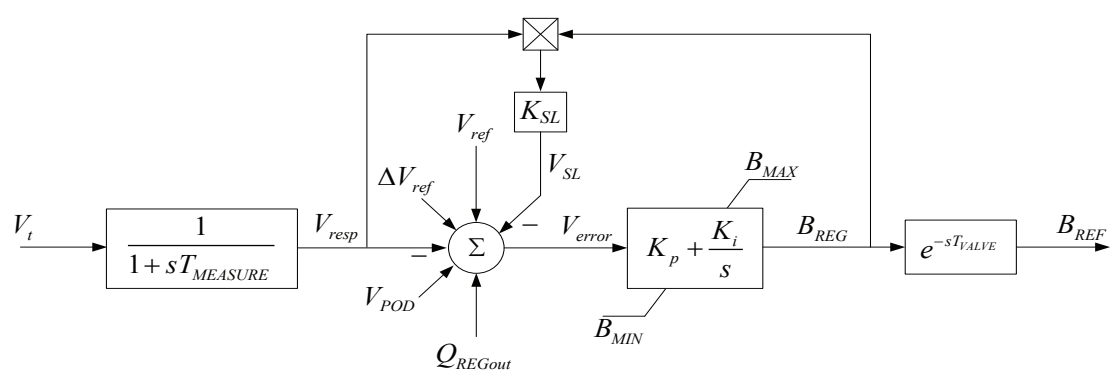

Figura 9. Diagrama a bloques del regulador de voltaje del CEV de CUT (De Oliveira et al., 2006) sirve para aplicar un pequeño escalón de voltaje para evaluar la respuesta al escalón del CEV. La referencia de voltaje del regulador de voltaje es $\mathrm{V}_{\mathrm{REF}}$. La variable $\mathrm{V}_{\mathrm{POD}}$ viene:

de una función para amortiguamiento de oscilaciones de potencia, en el caso del CEV de CUT no está implementada. La señal $Q_{\text {REGout }}$ viene de un regulador de susceptancia que actúa en condiciones de estado estacionario. La constante de tiempo de este regulador se encuentra en el orden de minutos por lo que no afecta el desempeño de CEV ante transitorios, en el CEV de CUT esta función está deshabilitada (De Oliveira et al., 2006). La Variable $\mathrm{K}_{\text {SLOPE }}$ es la pendiente de la característica V-I del CEV. La salida del punto de suma es la señal de error $\mathrm{V}_{\text {ERROR, }}$ la cual es aplicada al bloque del controlador proporcional integral, para el CEV del CUT el valor de la ganancia proporcional $\mathrm{K}_{\mathrm{p}}$ es cero, por lo que el controlador actúa como un integrador puro.
La ganancia integral es $\mathrm{K}_{\mathrm{i}}\left(\mathrm{K}_{\mathrm{i}}=400\right.$ para el CEV, se tomó el valor real que se tiene en el sistema de control), el control integral está acotado para trabajar dentro de los límites fijados por las constantes $\mathrm{B}_{\mathrm{MAX}}$ y $\mathrm{B}_{\mathrm{MIN}}$ (De Oliveira et al., 2006). Más información sobre sintonización de reguladores de voltaje de CEV se puede consultar en Mohan et al. (2002) y Taylor et al. (1994). Como se puede observar en la figura 9, la salida del regulador de voltaje es una señal de susceptancia por lo que se utilizó la ecuación (1) para calcular el valor del ángulo de disparo; para realizar el cálculo del valor de $\alpha$ a partir del valor de la susceptancia de referencia $\left(\mathrm{B}_{\mathrm{REF}}\right)$ se realizó una aproximación con polinomio utilizando Matlab.

\section{Simulación en Matlab/Simulink del CEV de CUT}

El programa visual en Simulink para simular el comportamiento del CEV instalado en la Subestación Culia-

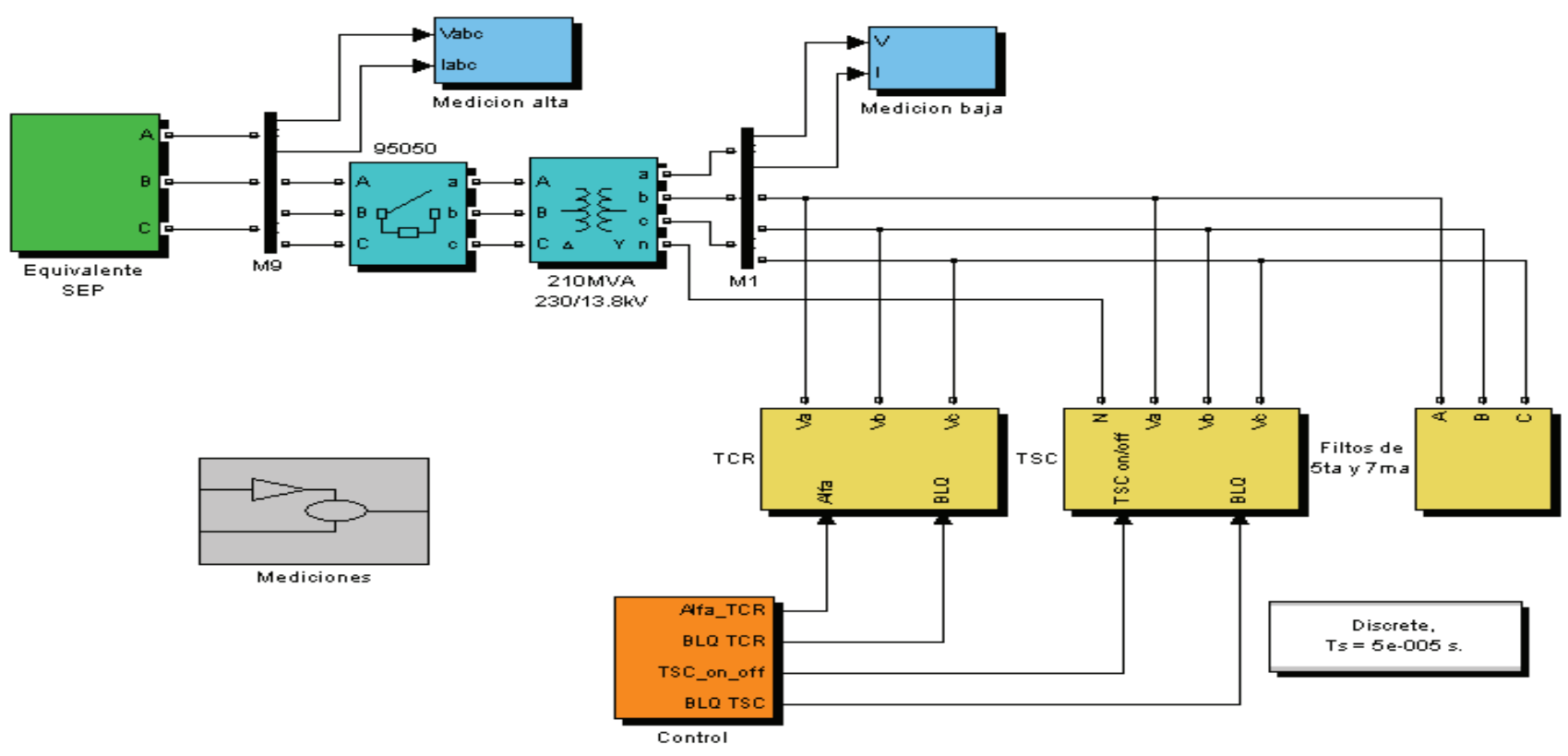

Figura 10. Programación del CEV de CUT en Simulink 
cán Tres se muestra en la figura 10. Para obtener el equivalente SEP se utilizó el programa ASPEN, en el cual están programados los principales elementos del Sistema Eléctrico Nacional y es utilizado por la Subgerencia de Protecciones de la Gerencia Regional para fines de simulación de fallas y ajuste de dispositivos de protección.

Los valores del equivalente SEP de la figura 10 son:

- Voltaje de fase a fase valor rms: $230 \mathrm{kV}$.

- Frecuencia de operación: $60 \mathrm{~Hz}$.

- Conexión interna de los devanados: estrella aterrizada.

- Nivel de corto circuito trifásico a voltaje base: 2285.1 MVA.

- El voltaje base del sistema: $230 \mathrm{kV}$.

- La relación X/R de la fuente: 3.76418 .

Este bloque tiene dos opciones, la primera es cuando se conoce el nivel de corto circuito con la relación $X / R$, que fue el utilizado en este caso, y la segunda, es cuando se conocen los valores de resistencia (R) e inductancia (L) equivalentes del sistema de potencia.

Para evaluar la característica estática del modelo Simulink del CEV de la figura 10 se introduce una rampa como voltaje de referencia $\mathrm{V}_{\text {ref }}$ como se muestra en la figura 11, la cual se ajusta para hacer variar gradualmente la susceptancia $B_{\text {ref }}$ de salida del regulador de voltaje desde su mínimo valor -1 hasta su valor máximo de 1 . La figura 11 muestra el resultado de la simulación donde en (b) se observa la variación del voltaje $\mathrm{V}_{\text {resp }}$ del lado primario $(230 \mathrm{kV})$ del CEV como respuesta a la susceptancia $B_{\text {ref }}$ del regulador de voltaje. En la figura 11 (c) se muestra la corriente del lado primario del CEV, la cual se considera negativa (inductiva: el CEV absorbe reactivos del SEP para bajar el voltaje) en el rango de tiempo de 1 a 3 segundos aproximadamente y se considera positiva (capacitiva: el CEV aporta reactivos al SEP para elevar el voltaje) en el rango de tiempo de 3 segundos en adelante.

En el CEV se usan los siguientes valores base: voltaje primario: $\mathrm{U}_{1 \mathrm{~b}}=230 \mathrm{kV}$; voltaje secundario: $\mathrm{U}_{1 \mathrm{~b}}=13.8$ $\mathrm{kV}$ y potencia aparente: $\mathrm{S}=100 \mathrm{MVA}$. Con estos productos se pueden calcular los valores base para la corriente del lado primario del transformador:

$I_{1 b}=\frac{S_{b}}{\sqrt{3} \cdot U_{1 b}}=\frac{100 \cdot 10^{3}}{\sqrt{3} \cdot 230}=251 \mathrm{~A}$

donde la susceptancia de respuesta del CEV, $\mathrm{B}_{\text {resp }}$, se calcula en por unidad de acuerdo a la ecuación:

Bresp $=\frac{\frac{\text { Icev }}{\text { Vresp }}}{B_{\text {cev_base }}} \quad B_{\text {cev_base }}=\frac{251 \mathrm{~A}}{230 \mathrm{kV}}$

Utilizando los resultados mostrados en la figura 11, se puede obtener la característica estática con el programa y compararla con la característica real del CEV de CUT. Como se puede observar en la figura 12 ambas características son similares.

Con el objetivo de obtener otras validaciones del programa se realizó una prueba donde el voltaje del SEP se ajustó a $233.8 \mathrm{kV}$, este ajuste se realiza en el bloque Equivalente SEP de la figura 10 y el voltaje de refe-

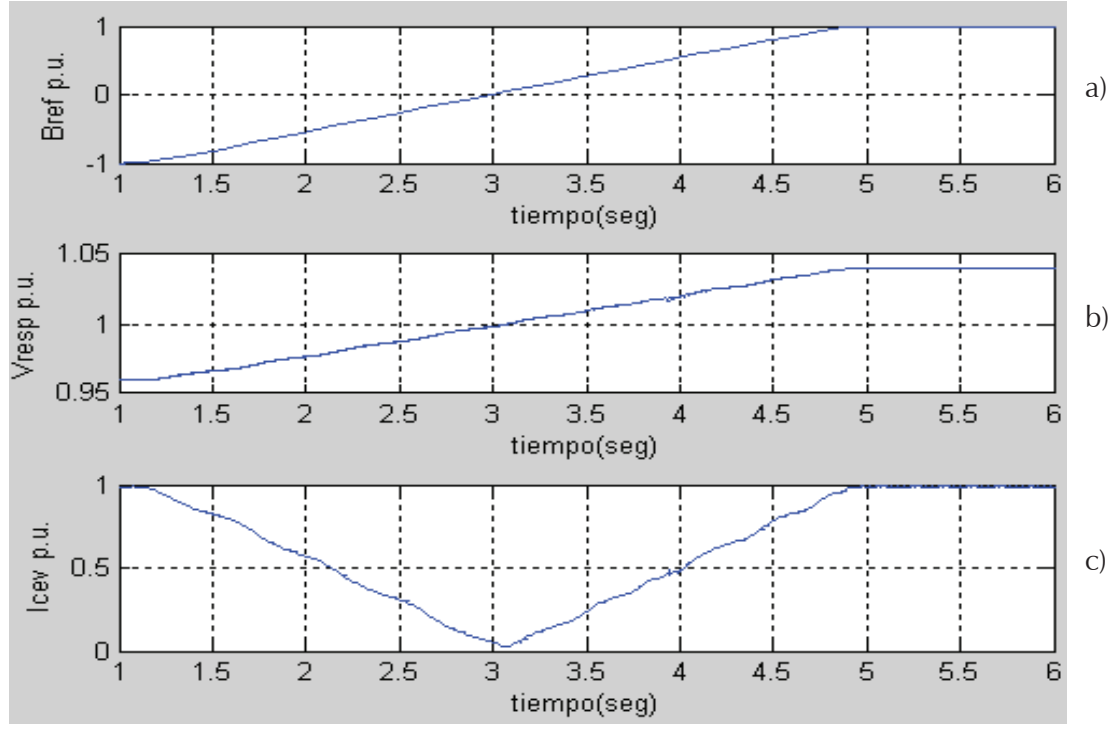

Figura 11. Resultado de la simulación al aplicar una rampa como $\mathrm{V}_{\text {ref }}$, a) susceptancia de salida $B_{\text {ref }}$ del regulador, b) respuesta de voltaje $\mathrm{V}_{\text {resp }}$ en el lado primario $230 \mathrm{kV}$ del CEV y c) corriente cev del lado primario 


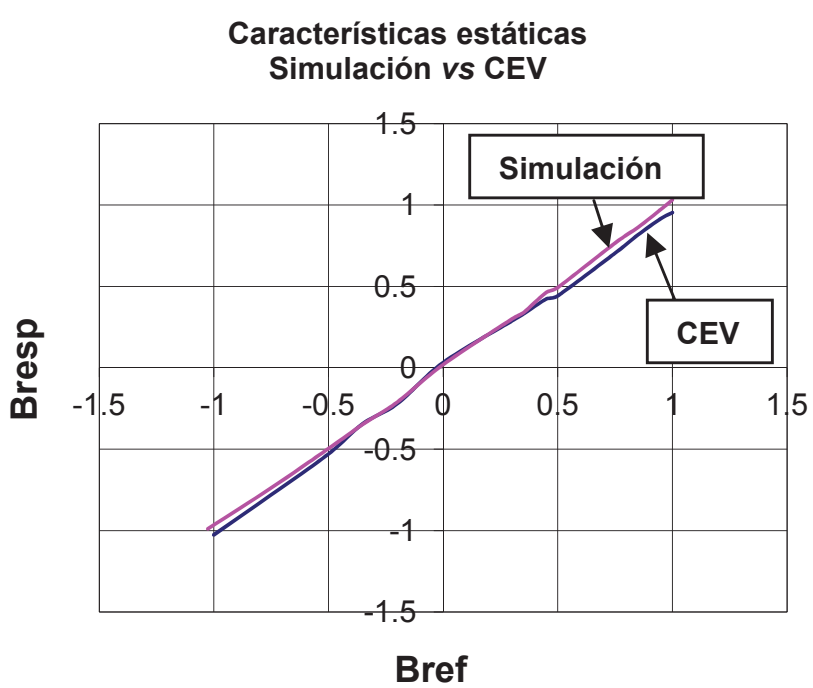

Figura 12. Característica estática obtenida del modelo en Simulink vs característica estática del CEV

rencia $\mathrm{V}_{\text {ref }}$ se ajustó a $233.45 \mathrm{kV}$ (1.015 p.u.). Los valores de ganancia y pendiente del control integral (figura 9), así como el escalón de voltaje aplicado son los siguientes: Ganancia $=350$, pendiente $=1 \%$, escalón $=-0.005$ p.u.

En la figura 13 se observa como $B_{\text {ref }}$ antes del escalón tiene un valor aproximadamente de cero; al aplicar el escalón de -0.005 actúa el regulador de voltaje y $B_{\text {ref }}$ va de 0 a -0.115 p.u., con lo cual la rama TCR incrementa su conducción de corriente (figura 13c) lo que provoca que el voltaje $\mathrm{V}_{\text {resp }}$ caiga $0.015-0.011=0.004$ p.u. (figura $13 b)$ en el lado de alta del CEV. La figura 14 corresponde a las pruebas realizadas durante la puesta en servicio del CEV de CUT con las mismas condiciones asumidas durante la simulación, al comparar las figuras 13 y 14 se puede ver que los valores de las variables son muy similares. En Coronel (2010) se realizaron más pruebas como la anterior, también con resultados satisfactorios.

\section{Simulación del CEV en la red troncal de la Zona Culiacán}

A continuación se muestra el programa visual en Simulink con la red troncal de la Zona Culiacán, donde se encuentra el CEV y se analiza su comportamiento ante cortocircuitos trifásicos, comparándolos con resultados obtenidos con el programa PSS/E. Resultados con cortocircuitos asimétricos se pueden encontrar en Coronel (2010). En la figura 15 se muestra el modelo en Simulink, donde se observan el equivalente de esa red troncal, pero solamente para las subestaciones CUT y GMD y las dos líneas de transmisión que las enlazan. El resto del sistema quedará incluido en los equivalentes de Thevenin conectados uno en el bus de $230 \mathrm{kV}$ de GMD (GMD-230) y el otro en el bus de $230 \mathrm{kV}$ de CUT (CUT230). El programa ASPEN permite obtener los equivalentes del SEP (en GMD y en CUT) y genera una línea virtual llamada línea equivalente del sistema (tabla 1).

Para las líneas de transmisión (93710 y 93730) se usó el modelo Simulink para líneas de transmisión con parámetros distribuidos. Para calcular estos parámetros distribuidos de las líneas de transmisión se utilizó la utilería de Matlab llamada Power_lineparam, donde los datos del conductor se tomaron de (Westinghouse, 1964). Para la línea equivalente se utilizó un modelo con parámetros concentrados cuyos valores se obtuvieron de los resultados obtenidos con el programa ASPEN, los valores por fase de la línea son: $R=5.3228 \Omega$, $L=$ $115.639 \mathrm{mH}$. La prueba consiste en aplicar un cortocircuito trifásico, con resistencia de falla de $26 \Omega$ directamente en el bus GMD-230. Primeramente se hace la

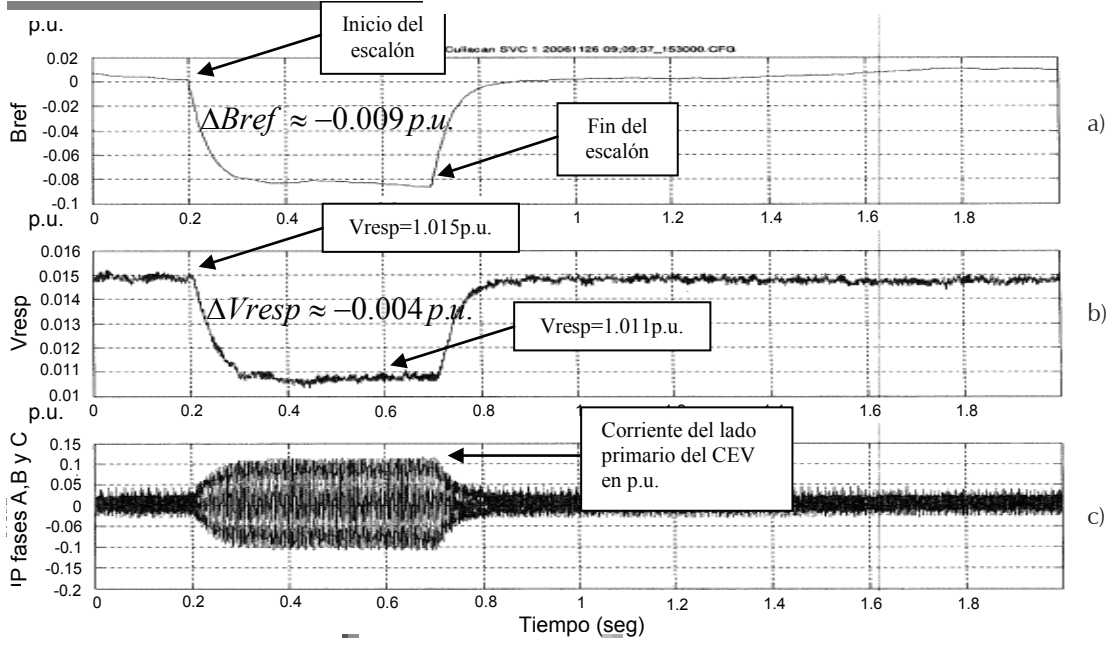

Figura 13. Resultados de la prueba con el modelo Simulink, a) susceptancia de salida $B_{\text {ref }}$ del regulador de voltaje en p.u., b) voltaje del lado de alta $(230 \mathrm{kV})$ del CEV y c) corrientes a, b y c del lado de alta del CEV en p.u. 


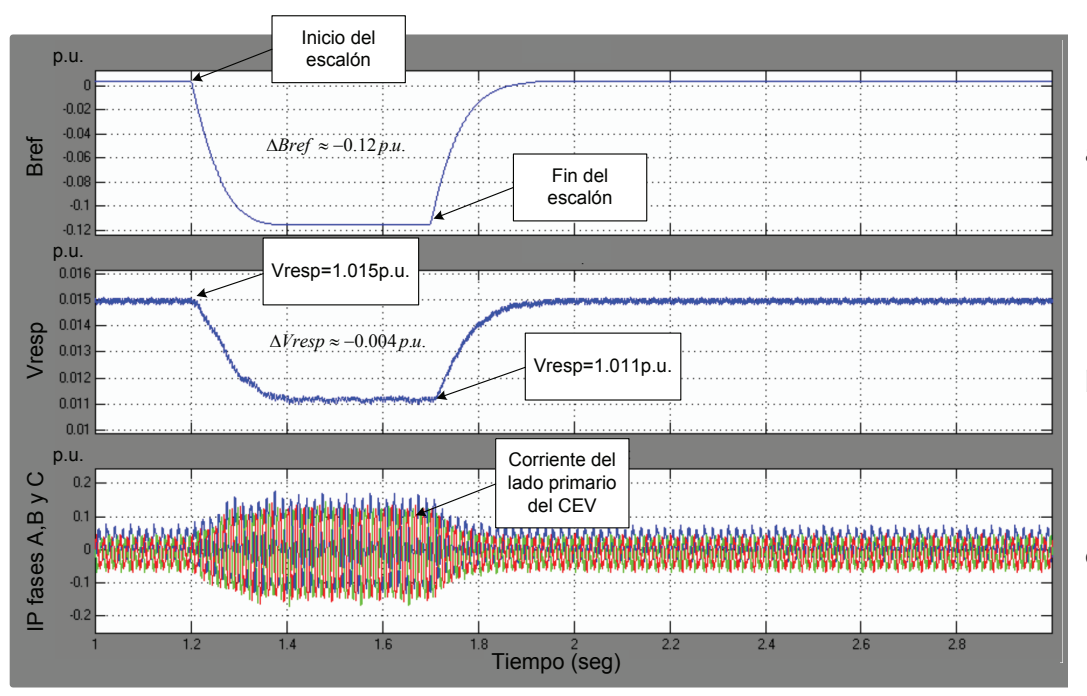

\begin{tabular}{ccc}
\hline & $\begin{array}{c}\text { Equivalente SEP en } \\
\text { GMD }\end{array}$ & $\begin{array}{c}\text { Equivalente } \\
\text { SEP en CUT }\end{array}$ \\
\hline Voltaje de fase a fase (rms) & $230 \mathrm{kV}$ & $234.15 \mathrm{kV}$ \\
Frecuencia de operación & $60 \mathrm{~Hz}$ & $60 \mathrm{~Hz}$ \\
Conexión interna de los devanados & estrella aterrizada & estrella aterrizada \\
Nivel de corto circuito trifásico a & $1069.9 \mathrm{MVA}$ & $1588.7 \mathrm{MVA}$ \\
voltaje base & $230 \mathrm{kV}$ & $230 \mathrm{kV}$ \\
Voltaje base del sistema & 8.6571 & 13.3341 \\
Relación X/R de la fuente & & \\
\hline
\end{tabular}

a)

b)

Figura 14. Resultado de la prueba durante la puesta en servicio,

a) susceptancia de salida $B_{\text {ref }}$ del regulador en p.u., b) voltaje del lado de alta en p.u., y c) corrientes de las fases a, b y c del lado de alta del CEV en p.u.

Tabla 1. Valores del equivalente del sistema en las subestaciones GMD y CUT obtenidos con ASPEN

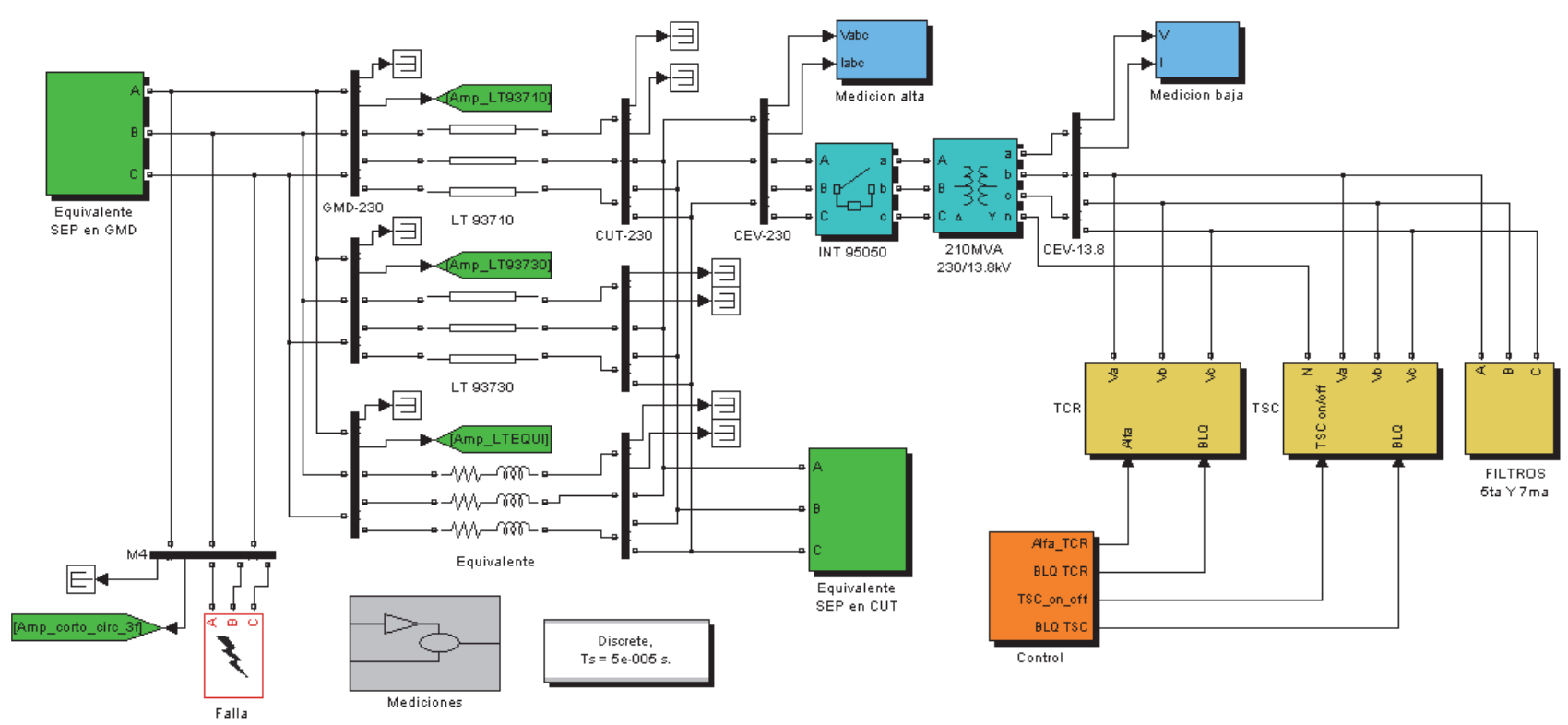

Figura 15. Programa gráfico en Simulink para obtener la respuesta del CEV ante una falla trifásica en GMD-230 
prueba utilizando el programa PSS/E que utiliza la CFE para análisis de SEP obteniéndose los resultados que permiten posteriormente reproducir la falla en el programa mostrado en la figura 15 con las mismas condiciones iniciales. De esta forma se comparan los resultados, con lo cual se puede realizar una validación del programa en Simulink.

En la figura 16 se muestran los resultados obtenidos con el PSS/E, donde un instante antes de que ocurra una falla trifásica en el bus de GMD-230, el CEV aporta
-12.9 MVARS al sistema. Cuando ocurre la falla trifásica con condiciones tales que el voltaje en el bus de CUT se abate a 0.786 p.u., el CEV aporta 64.63 MVARS durante el periodo de la falla, y posteriormente aporta 113.06 MVARS, tratando de regresar a la condición que tenía antes de la falla.

Para la simulación de la falla trifásica con el modelo Simulink se busca reproducir el resultado obtenido con las simulaciones en el programa PSS/E. En la figura 16 se observa que antes de la falla el voltaje en el bus de

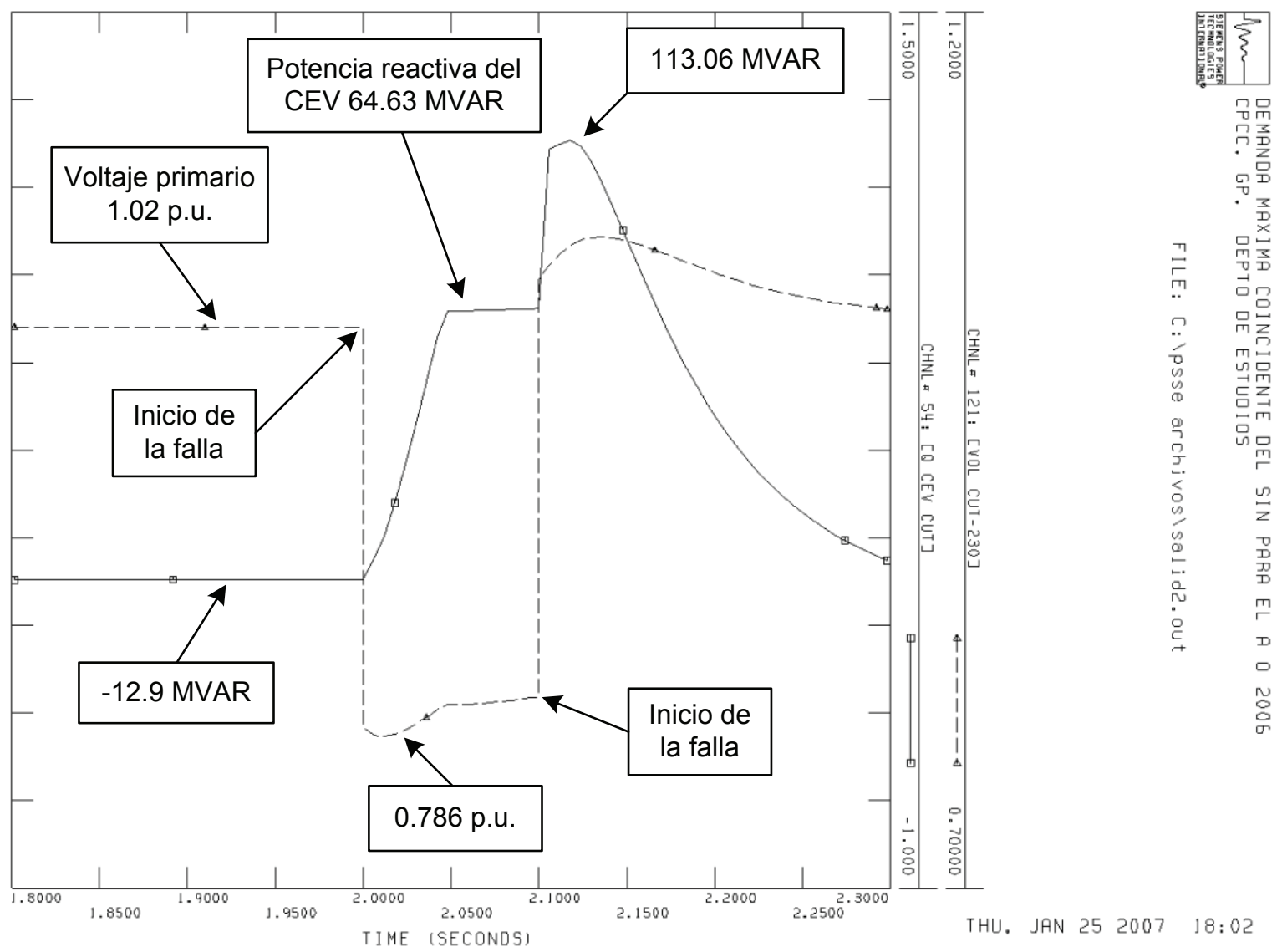

Figura 16. Comportamiento del CEV ante una falla trifásica en el bus GMD-23, usando el programa PSS/E

Tabla 2. Valores introducidos al modelo Simulink para la prueba de corto circuito trifásico en el bus GMD-230

\begin{tabular}{|c|c|c|c|c|c|c|}
\hline $\begin{array}{l}\text { Equivalente SEP } \\
\text { en GMD }(\mathrm{kV})\end{array}$ & $\begin{array}{c}\text { Equivalente SEP } \\
\text { en } \\
\text { CUT }(\mathrm{kV})\end{array}$ & $\begin{array}{c}\text { Referencia } \mathrm{V}_{\text {ref }} \text { del } \\
\text { regulador } \\
(\mathrm{kV})\end{array}$ & $\begin{array}{l}\text { Ganancia del } \\
\text { regulador } 1 / \mathrm{s}\end{array}$ & $\begin{array}{l}\text { Pendiente del } \\
\text { regulador \% }\end{array}$ & $\begin{array}{l}\text { Resistencia de falla } \\
\mathrm{R}_{\mathrm{on}} \Omega\end{array}$ & $\begin{array}{l}\text { Duración } \\
\text { de la fallas }\end{array}$ \\
\hline 230 & 234.15 & 234.5 & 400 & 0.01 & 26 & 0.1 \\
\hline
\end{tabular}




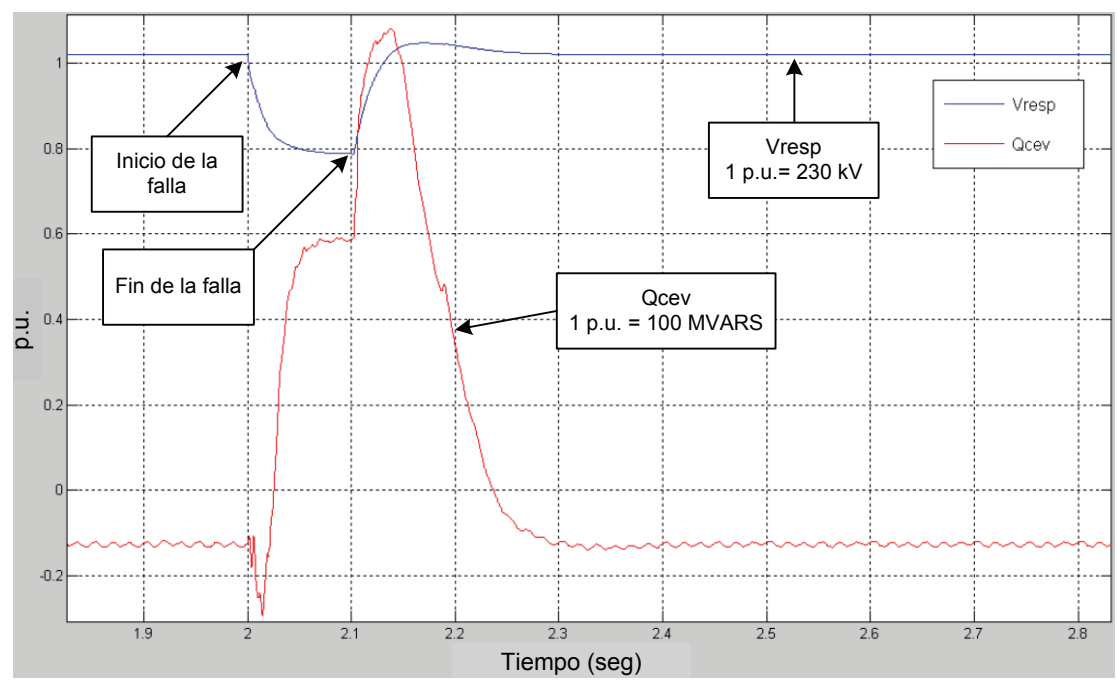

Figura 17. Comportamiento del CEV ante una falla trifásica (resistencia de falla $26 \Omega$ ) en el bus GMD-230, usando el modelo Simulink
CUT-230 es de aproximadamente 1.02 p.u., y la potencia reactiva del CEV es de aproximadamente -12.9 MVARS. Para lograr estas condiciones iniciales con el programa en Simulink del CEV de la figura 15, se le introdujeron los parámetros mostrados en la tabla 2.

La figura 17 muestra el resultado de la simulación del programa Simulink de la figura 15 al aplicar una falla de corto circuito trifásico en el bus GMD-230. Comparando los resultados de las figuras 16 y 17 se puede apreciar que los valores de las variables son similares.

\section{Conclusiones}

La característica estática del CEV obtenida con el programa Simulink es muy similar a la que se logró durante las pruebas de puesta en servicio del CEV. Los resultados de las pruebas de respuesta al escalón son comparables a los obtenidos durante las pruebas de puesta en servicio. Durante estas simulaciones se observó que es difícil simular las condiciones exactas en las cuales se encontraba el SEP cuando se llevaron a cabo las pruebas de respuesta al escalón, ya que el equivalente de SEP obtenido con el ASPEN y programado en Simulink no contempla las condiciones del sistema de potencia al momento de las pruebas de puesta en servicio del compensador bajo estudio, no obstante, se logró aproximar con una precisión aceptable dichas condicio- nes. La respuesta del modelo Simulink del CEV ante una falla trifásica ofrece resultados similares a los obtenidos con el PSS/E, lo cual permite señalar que con el programa de Simulink se obtienen resultados satisfactorios. La contribución más importante del trabajo es proporcionar a la Gerencia Regional de Transmisión Noroeste de una herramienta útil para el análisis de la operación del Compensador Estático de VARS instalado en la Subestación Culiacán Tres del Estado de Sinaloa, además permite el análisis de fallas que se puedan presentar en la red troncal de la zona de Culiacán. El trabajo podría ser extendido a otros CEV del país.

\section{Referencias}

Braegger R. FACTS e a Estabilidade, Dinâmica e Estabilidade de Sistemas Eléctricos, tesis (maestría), FEUP, Mayo 2005.

Coronel L.E. Modelado del compensador estático de VARS de CUT para análisis de transitorios electromagnéticos, tesis (maestría), Instituto Politécnico Nacional, México, octubre de 2010.

De Oliveira M. y Larsson D. Main Component Design-Culiacán SVC, ABB Power Technologies, Document Number, 1JNR 100006-750, septiembre de 2005.

De Oliveira M. y Larsson D. SVC Model in PSS/E. ABB Power Technologies. Document Number: 1JNR100008-169, 2006.

Hingorani N.G. y Gyugyi L. Understanding FACTS, Concepts and Technology of Flexible AC Transmission Systems, IEEE PRESS, 2000. 
Mohan M.R. y Varma R.K. Thyristor-Based FACTS Controllers for Electrical Transmission Systems, IEEE Series on Power Engineering, Wiley Inter-Science, 2002.

Pettersson L. y Hedman J. Descripción funcional del sistema de control, ABB Power Technologies, documento número 1jnr100005-413, septiembre, 2005.

Taylor C., Scott G., Hammad A., Wong W., Osborn D., Ramos A.J.P., Johnson B., Nabb D.M., Arabi S., Martin D, Thanawala H. L., Luini J., Gonzalez R. y Concordia C. Static VARS Compensator Models for Power Flow and Dynamic Performance Simulation. IEEE Transactions on Power Systems, volumen 9 (número 1), febrero 1994.

Westinghouse Electric Corporation. Electrical Transmission and Distribution Reference Book, 4a. ed., 1964.

\begin{abstract}
Este artículo se cita:
Citación estilo Chicago

Coronel-Mercado, Luis Enrique, Jaime José Rodríguez-Rivas, Pedro Francisco Huerta-González. Simulación del compensador estático de VAR de la subestación Culiacán Tres para el análisis de transitorios. Ingeniería Investigación y Tecnología, XIV, 03 (2013): 423-436.

Citación estilo ISO 690

Coronel-Mercado L.E., Rodríguez-Rivas J.J., Huerta-González P.F. Simulación del compensador estático de VAR de la subestación Culiacán Tres para el análisis de transitorios. Ingeniería Investigación y Tecnología, volumen XIV (número 3), julio-septiembre 2013: 423-436.
\end{abstract}

\section{Semblanza de los autores}

Luis Enrique Coronel-Mercado. Ingeniero electrónico por el Instituto Tecnológico de Culiacán, México (1996). Trabaja en la Comisión Federal de Electricidad desde el año de 1997, donde actualmente ocupa el cargo de jefe del Departamento de Control de la Subárea de Transmisión Culiacán. Terminó su maestría en ciencias en ingeniería eléctrica en la Sección de Estudios de Posgrado e Investigación (SEPI), de la ESIME-Zacatenco del Instituto Politécnico Nacional en 2010.

Jaime José Rodríguez-Rivas. Es ingeniero electricista egresado del la Universidad Central de Las Villas (UCLV), Cuba en 1980. Obtuvo el grado de maestro en ciencias en 1987 y el de doctor en ciencias en 1991, ambos en el Instituto Energético de Moscú. Trabajó como profesor en la UCLV desde 1980 hasta 1994. Desde 1994 a la fecha trabaja como profesor investigador en la SEPI, ESIMEZ, del Instituto Politécnico Nacional. Es miembro de la IEEE.

Pedro Francisco Huerta-González. Ingeniero electricista de la Escuela Superior de Ingeniería Mecánica y Eléctrica del Instituto Politécnico Nacional (1997). Obtuvo el grado de maestro en ciencias en ingeniería eléctrica opción control en la Sección de Estudios de Posgrado e Investigación, ESIME Zacatenco del Instituto Politécnico Nacional en 2004. Actualemnte es profesor titular A en la carrera de ingeniería en control y automatización. 